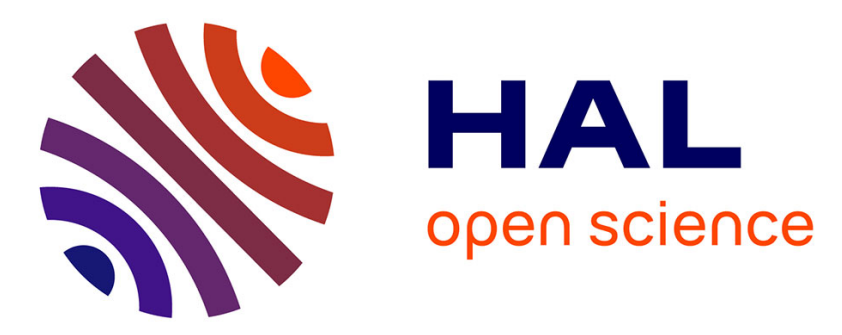

\title{
Shape Measurement Using a New Multi-step Stereo-DIC Algorithm That Preserves Sharp Edges
}

J. Harvent, Benjamin Coudrin, L. Brèthes, Jean-José Orteu, Michel Devy

\section{To cite this version:}

J. Harvent, Benjamin Coudrin, L. Brèthes, Jean-José Orteu, Michel Devy. Shape Measurement Using a New Multi-step Stereo-DIC Algorithm That Preserves Sharp Edges. Experimental Mechanics, 2015, 55 (1), pp.167-176. 10.1007/s11340-014-9905-z . hal-01169750

\section{HAL Id: hal-01169750 \\ https://imt-mines-albi.hal.science/hal-01169750}

Submitted on 30 Jun 2015

HAL is a multi-disciplinary open access archive for the deposit and dissemination of scientific research documents, whether they are published or not. The documents may come from teaching and research institutions in France or abroad, or from public or private research centers.
L'archive ouverte pluridisciplinaire HAL, est destinée au dépôt et à la diffusion de documents scientifiques de niveau recherche, publiés ou non, émanant des établissements d'enseignement et de recherche français ou étrangers, des laboratoires publics ou privés. 
Experimental Mechanics manuscript No.

(will be inserted by the editor)

1 Shape Measurement Using a New Multi-Step Stereo-DIC

2 Algorithm That Preserves Sharp Edges

Jacques Harvent • Benjamin Coudrin • Ludovic

4 Brèthes · Jean-José Orteu •

5 Michel Devy

6 Received: date / Accepted: date

7 Abstract Digital Image Correlation is widely used for shape, motion and deforma-

8 tion measurements. Basically, the main steps of 3D-DIC for shape measurement

9 applications are: off-line camera calibration, image matching and triangulation.

10 The matching of each pixel of an image to a pixel in another image uses a so-

11 called subset (correlation window). Subset size selection is a tricky issue and is a trade-off between a good spatial resolution, achieved with small subsets that preserve image details, and a low displacement uncertainty achieved with large subsets that can smooth image details.

In this paper, we present a new multi-step DIC algorithm specially designed for measuring the 3D shape of objects with sharp edges. With this new algorithm

J. Harvent

NOOMEO, 425 rue Jean Rostand, F-31670 Labège, France E-mail: jacques.harvent@gmail.com

B. Coudrin

CNRS ; LAAS ; 7 avenue du colonel Roche, F-31400 Toulouse, France

Université de Toulouse ; LAAS ; F-31400 Toulouse, France E-mail: bcoudrin@laas.fr

L. Brèthes

NOOMEO, 425 rue Jean Rostand, F-31670 Labège, France E-mail: ludovic.brethes@noomeo.eu

J.-J. Orteu

Université de Toulouse ; Mines Albi ; ICA (Institut Clément Ader) ; Campus Jarlard, F-81013

Albi, France E-mail: jean-jose.orteu@mines-albi.fr

M. Devy

CNRS ; LAAS ; 7 avenue du colonel Roche, F-31400 Toulouse, France

Université de Toulouse ; LAAS ; F-31400 Toulouse, France E-mail: michel.devy@laas.fr 
an accurate $3 \mathrm{D}$ reconstruction of the whole object, including sharp edges that are preserved, can be achieved.

Keywords Shape Measurement · Stereovision · Digital Image Correlation (DIC) · Edge Preservation · Accurate 3D reconstruction.

\section{Introduction}

The problem of improving the accuracy of the 3D reconstruction of a complex object is addressed by using a DIC-based shape measurement technique. The DIC technique requires choosing a correlation criterion, the size of the correlation window (also called subset) and the window transformation model (subset shape function). The choice of the subset size is a tricky issue [1-4] and is a trade-off between a good spatial resolution, achieved with small subsets that preserve image details, and a low displacement uncertainty achieved with large subsets that can smooth image details (see figure 1 and table 1 ).

\begin{tabular}{|c|c|c|}
\hline subset size & displacement uncertainty & shape details \\
\hline small & high & preserved \\
\hline large & low & smoothed \\
\hline
\end{tabular}

Table 1 Trade-off between displacement uncertainty and shape details preservation

In order to increase the accuracy of the shape reconstruction, several authors have proposed to use a multi-view stereo method that exploits a large number of images [5-8] and that allows using a small subset size.

In this paper, we address the problem of providing an accurate $3 \mathrm{D}$ reconstruction of an object by using the classical two-views 3D-DIC technique.

In the classical DIC-based matching technique, discontinuities (e.g. cracks in 2D-DIC-based displacement/strain measurements, sharp edges in stereo-DICbased shape measurements) are difficult to handle and several authors have proposed new DIC formulations specially designed for DIC matching in presence of 

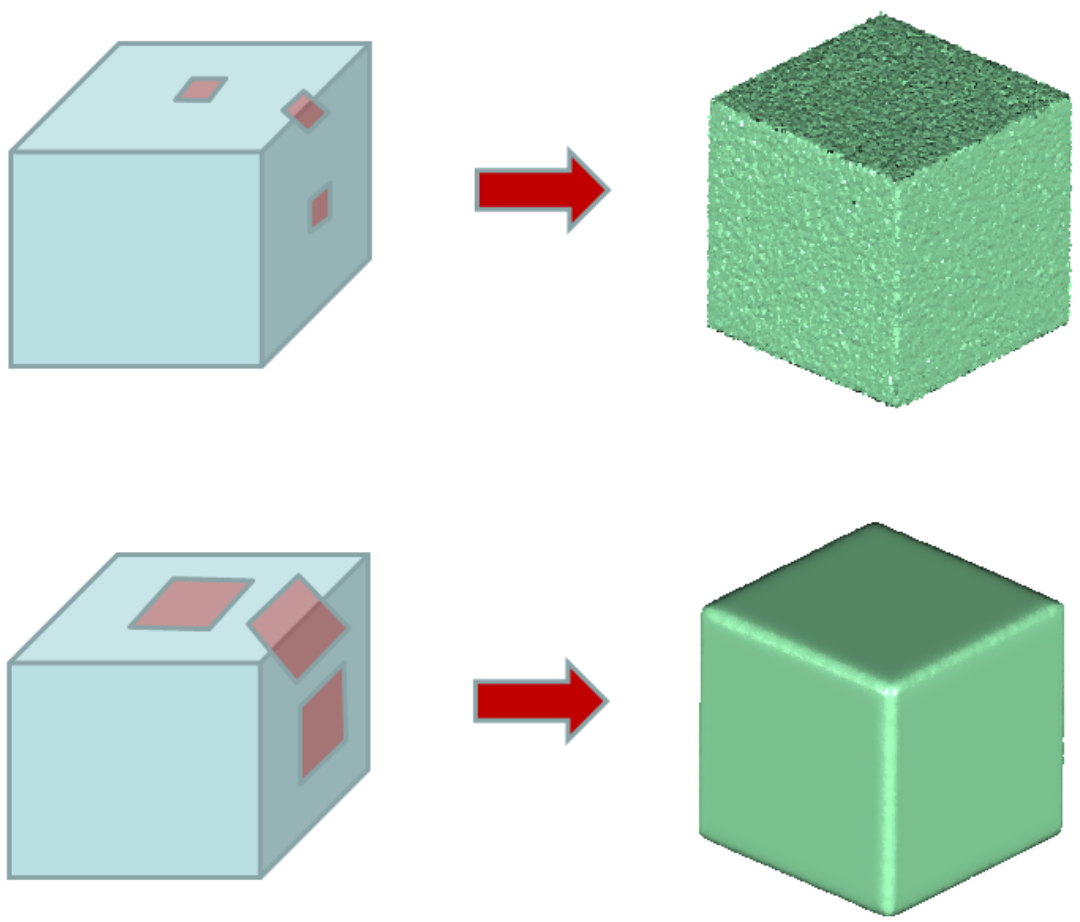

Fig. $13 \mathrm{D}$ reconstruction using a small subset (top) and 3D reconstruction using a large subset (bottom)

discontinuities [9-12]. Most of these works deal with 2D discontinuities and there is no work to the authors knowledge that deals with discontinuities for 3D-DIC.

The extraction of edges can be performed after the 3D shape measurement, using a 3D mesh like in [13]. An analysis of the 3D mesh allows a fine extraction of the surface discontinuities, then of the edges. In our method, sharp edges are obtained using DIC and are extracted directly from the 3D points cloud generated by the stereo-DIC-based method, without building a 3D mesh.

We propose a new DIC method, based on a new correlation criterion and a new window transformation model, specially designed to preserve the sharp edges that can be present on many machined objects. 
2 Overview

The presented method is an extension to the standard stereo-vision 3D reconstruction. It aims to refine the reconstruction accuracy for points near sharp edges. These points are generally badly estimated due to an over-smoothing effect. Standard stereo-vision uses the approximation that the observed surface is locally continuous and almost planar. This assumption becomes false near sharp edges causing a false estimation of the surface. Due to the planar approximation, standard stereo-vision tends to measure edges as curves. To achieve the sharp edges preservation, our proposed method performs an iterative refinement of the areas where the planar approximation fails. It starts from an initial 3D reconstruction of an object.

The initial 3D reconstruction is obtained using a standard stereo-vision method and is briefly presented in section 4 . It provides a $3 \mathrm{D}$ point cloud representation of the surface, and equally computes normals and confidence values of the measured points.

From this initial reconstruction, our edge-optimized method refines iteratively the position of the edges. Our algorithm is composed of three steps.

Firstly, it detects regions with high curvature. These regions are considered candidates to the edge refinement. This allows us to reduce the number of points which need to be processed. It is presented in section 5 .

Secondly, the algorithm computes a first estimate of the possible location of the edges for each area (section 6).

Finally, every considered point is estimated using our new "bi-plane" model. This model is based on the two planes which compose an edge, and is refined minimizing a correlation criterion. It is presented in section 7 .

The proposed strategy to improve the accuracy near sharp edges is itself based on a two-planes assumption, i.e. the neighborhood of every point on the object surface can be approximated by two planes. In some situations (chamfers perceived from a far viewpoint), this assumption can be violated. An additional step is 
necessary to validate the results. The residual error from the standard stereo-vision method and the residual error from our new edge-optimized method are compared.

The reconstruction which provides lower error is chosen. This ensures that high curvature areas not representing actual sharp edges would not be "sharpened" by error.

\section{Problem formulation and notation}

We consider a stereo-vision system with two digital cameras rigidly attached. We associate to each camera an euclidean coordinate frame. Respectively $\mathcal{C}_{0}$ and $\mathcal{C}_{1}$ for the principal camera and the secondary one: 3D points are reconstructed in the reference frame of the principal camera. Every camera is calibrated using the method from [14], modified to allow automatic initialization; the rigid transformation between the reference frames of the two cameras is also estimated using the standard stereo calibration toolbox. Images are considered distortion free i.e. the images are provided by a perfect distortion free system or the images have been corrected from their distortion using the calibration parameters. Moreover, images and transformations are considered in the epipolarly rectified space. This transformation between the cameras is noted $\left[\mathbf{I}_{3 \times 3} \mid \mathbf{t}_{\mathcal{C}_{1} \mathcal{C}_{0}}\right] \in S E(3)$, where $\mathbf{I}_{3 \times 3}$ is a $3 \times 3$ identity matrix and $\mathbf{t}_{\mathcal{C}_{1} \mathcal{C}_{0}}$ is the translation between the origins of the camera frames, with only the baseline along the $X$ axis.

The stereo-imaging process can then be represented using a linear model relating a $3 \mathrm{D}$ point in the world (reference) coordinate frame $\mathbf{m}_{\mathcal{W}}=(X, Y, Z, 1)^{T}$ to two image points $\mathbf{m}_{\mathcal{I}_{0}}=\left(s x_{0}, s y, s\right)^{T}$ and $\mathbf{m}_{\mathcal{I}_{1}}=\left(s x_{1}, s y, s\right)^{T}$ the projections of $\mathbf{m}_{\mathcal{W}}$ in, respectively, the image coordinate frames $\mathcal{I}_{0}$, related to the camera $\mathcal{C}_{0}$, and $\mathcal{I}_{1}$, related to the camera $\mathcal{C}_{1}$. Due to the epipolar rectification, images of the same $3 \mathrm{D}$ points lie on the same image line and have then the same $y$ coordinate [15]. 
The imaging process can be formulated as follow.

$$
\begin{aligned}
& \mathbf{m}_{\mathcal{I}_{0}}=\mathbf{K}_{0}\left[\mathbf{R}_{\mathcal{C}_{0} \mathcal{W}} \mid \mathbf{t}_{\mathcal{C}_{0} \mathcal{W}}\right] \mathbf{m}_{\mathcal{W}} \\
& \mathbf{m}_{\mathcal{I}_{1}}=\mathbf{K}_{1}\left[\mathbf{I}_{3 \times 3} \mid \mathbf{t}_{\mathcal{C}_{1} \mathcal{C}_{0}}\right]\left[\mathbf{R}_{\mathcal{C}_{0} \mathcal{W}} \mid \mathbf{t}_{\mathcal{C}_{0} \mathcal{W}}\right] \mathbf{m}_{\mathcal{W}}
\end{aligned}
$$

where $\mathbf{K}_{0}$ and $\mathbf{K}_{1}$ are the calibration matrices of, respectively, cameras $\mathcal{C}_{0}$ and $\mathcal{C}_{1}$, formed from the cameras independent intrinsic parameters, and $\left[\mathbf{R}_{\mathcal{C}_{0} \mathcal{W}} \mid \mathbf{t}_{\mathcal{C}_{0} \mathcal{W}}\right] \in$ $S E(3)$ is the transformation between the world frame $\mathcal{W}$ and the principal camera frame $\mathcal{C}_{0}$. This transformation is called the pose of the system.

\section{Initial reconstruction}

Standard stereo-vision reconstruction is the process of finding the $3 \mathrm{D}$ point coordinates $\mathbf{m}_{\mathcal{W}}$ from its image points $\mathbf{m}_{\mathcal{I}_{0}}$ and $\mathbf{m}_{\mathcal{I}_{1}}$.

A common stereo-reconstruction approach is to try to match pixels in the pair of images using the luminance information of the images. This is achieved by determining the point $\mathbf{m}_{\mathcal{I}_{1}}$ matching the point $\mathbf{m}_{\mathcal{I}_{0}}$. This is done by observing and matching luminance information in both images, $\mathcal{I}_{0}$ and $\mathcal{I}_{1}$. Luminance functions are noted $\mathbf{I}_{0}(\mathbf{p})$ and $\mathbf{I}_{1}(\mathbf{p})$, respectively for images $\mathcal{I}_{0}$ and $\mathcal{I}_{1}$. To avoid ambiguities, the matching point is found by comparing local appearance around the pixel $\mathbf{m}_{\mathcal{I}_{0}}$. Surface can be approximated locally by tangent planes to observed points. The matching process is the similarity measurement of the projections of an area of this approximated plane in the images. The transformation relating the projections in each image is an homography (figure 2).

From [8] we have the following homography formulation: 


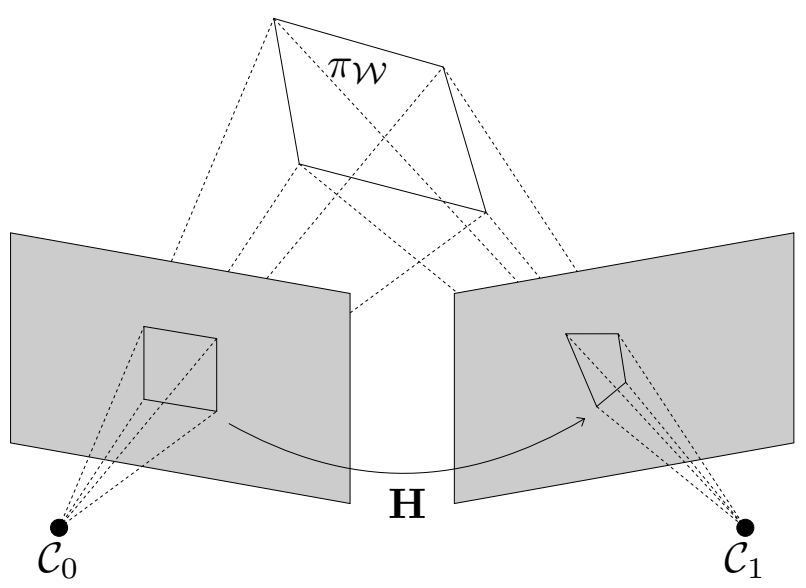

Fig. 2 An homography is a projective transformation relating the projections of a plane in two images

$$
\begin{aligned}
\mathbf{H} & =\mathbf{K}_{1}\left[\mathbf{I}_{3 \times 3} \mid \mathbf{t}_{\mathcal{C}_{1} \mathcal{C}_{0}}\right] \mathbf{S K}_{0}^{-1} \\
\mathbf{S} & =\left[\begin{array}{ccc}
-1 & 0 & 0 \\
0 & -1 & 0 \\
0 & 0 & -1 \\
n_{x} & n_{y} & n_{z}
\end{array}\right]
\end{aligned}
$$

where $\left(n_{x}, n_{y}, n_{z}\right)^{T}$ is the normal of the tangent plane to the surface at $\mathbf{m}_{\mathcal{W}}$.

The structure of the scene, from two images acquired at the same time, is obtained by minimizing dissimilarities between the luminance function $\mathbf{I}_{0}(\mathbf{p})$ in the region $W$ of image $\mathcal{I}_{0}$ and the luminance function $\mathbf{I}_{1}(\mathbf{p})$ in the transformed region $\mathbf{H}(W)$ of image $\mathcal{I}_{1}$. If we note $\mathbf{x}=\left(n_{x}, n_{y}, n_{z}\right)$, we try to find

$$
\underset{\mathbf{x}}{\operatorname{argmin}} \sum_{i \in W}\left(\mathbf{I}_{0}\left[\mathbf{p}_{\mathcal{I}_{0}}^{i}\right]-\mathbf{I}_{1}\left[\mathbf{H}_{i}\left(\mathbf{p}_{\mathcal{I}_{0}}^{i}, \mathbf{x}\right)\right]\right)^{2}
$$

Equation (5) introduces the minimization criterion used to find, for a given point $m_{I_{0}}$, the matched point $m_{I_{1}}$, using only the parameters of the tangent plane 


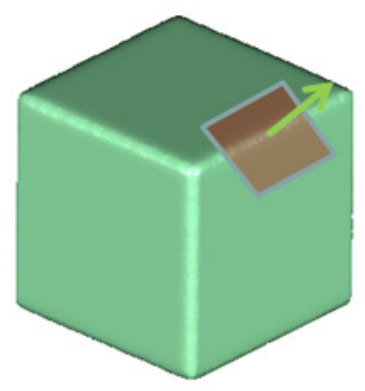

Fig. 3 The plane model does not fit the surface along the edges

defined by the correlation of two pixel regions in two stereo images. This problem can be solved using standard non-linear least-squares methods.

This approach is valid and has proved to work well on nearly planar surfaces. The assumption that the observed region $W$ is a plane tangent to surface at $\mathbf{m}_{\mathcal{W}}$ introduces an approximation resulting in a surface smoothing (figure 3).

The reconstruction process on very non-planar surfaces is a trade-off between accuracy and stability by tuning the size of the region $W$.

Particularly, when the region $W$ contains sharp edges, the tangent planeinduced homography is not a valid model. It tends to reduce the discontinuity of the surface. Our method proposes to correct the result of the process we have described by detecting sharp edges in the model and re-correlating the erroneous points using a "bi-plane" model.

\section{Edge detection}

The aim of our proposed method is to refine the initial reconstruction we have presented to allow reconstruction of sharp edges. Only the points near sharp edges 
have to be refined. To avoid processing unnecessary points, we add an edge detection step.

Since the method we have described tends to smooth discontinuities due to the approximation of the local surface, edges are reconstructed as short arc curves. For each $3 \mathrm{D}$ point $\mathbf{p}_{0}$, with its normal $\mathbf{n}_{0}$, let us consider the neighborhood $W$ consisting of nearest points $\mathbf{p}_{i} \in W$ around $\mathbf{p}_{0}$. Each point $\mathbf{p}_{i}$ is associated with its normal $\mathbf{n}_{i}$. We compute the mean normal $\overline{\mathbf{n}}$, the mean $\mu$ and the standard deviation $\sigma$ of the dot products to this mean normal in $W$.

$$
\begin{aligned}
\overline{\mathbf{n}} & =\frac{1}{\left\|\sum_{i \in W\left(\mathbf{p}_{0}\right)} \mathbf{n}_{i}\right\|} \sum_{i \in W\left(\mathbf{p}_{0}\right)} \mathbf{n}_{i} \\
\mu & =\frac{1}{\operatorname{card}\left(W\left(\mathbf{p}_{0}\right)\right)} \overline{\mathbf{n}} \cdot \sum_{i \in W\left(\mathbf{p}_{0}\right)} \mathbf{n}_{i} \\
\sigma & =\sqrt{\frac{1}{\operatorname{card}\left(W\left(\mathbf{p}_{0}\right)\right)} \sum_{i \in W\left(\mathbf{p}_{0}\right)}\left(\overline{\mathbf{n}} \cdot \mathbf{n}_{i}-\mu\right)^{2}}
\end{aligned}
$$

Points on short arc curves are identified from high deviation regions to ensure that we select regions with high curvature. The standard deviation $\sigma$ of the dot products represents the variability of the normal distribution in $W$ and is then used as the deviation measurement.

The detection process consists in thresholding the standard deviation $\sigma$. The criterion is used to build a selection function $\Pi(\mathbf{p})$.

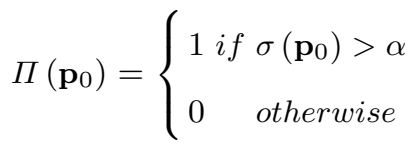

where $\alpha$ is the threshold, allowing to be more or less selective on the edge detection. A higher threshold will reject more points, increasing the probability of non-detection. A lower threshold will result in a less selective behavior, increasing the probability to select non-edges points. 


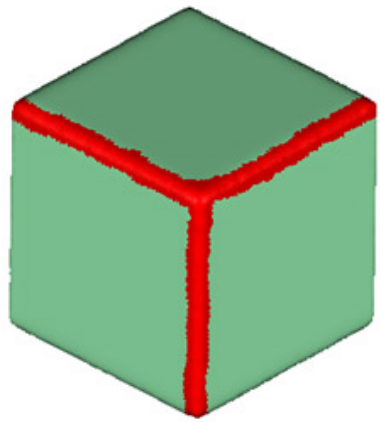

Fig. 4 Selection (red) of the edges of the cube

The edge selection process allows us to save time by processing less non-edge points. The results of our method is not likely to improve points that do not lie on edges. We discuss in section 7.2 this matter and how we deal with wrongly selected points.

\section{Initial edge estimation}

An initial estimate of the edge model is computed for each selected point, and this estimate is refined later (as described in section 7). The edge model consists of two mean normals each of which describes one face of the edge. In order to compute these two normals, we consider a region around the edge and then we classify the points and their normal in two groups using a modified k-means clustering algorithm. In section 5 the standard deviation $\sigma$ of the dot products to the mean normal was computed for each point. This value is introduced in the clustering process, in order to filter points with a large $\sigma$. A plane is then fitted to each cluster of points. The result provides the two mean normals. 


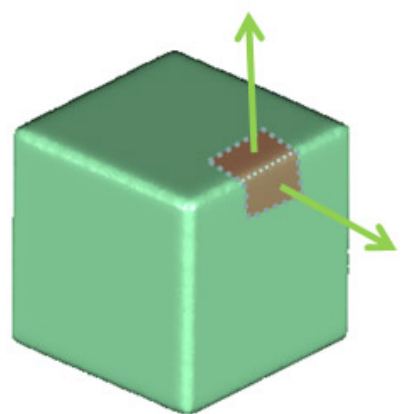

Fig. 5 The "bi-plane" model is represented by two planes defined by their normals (green)

The intersection can be expressed as Plücker axial coordinates:

$$
\mathcal{L}=\left[\mathbf{L}_{23}: \mathbf{L}_{31}: \mathbf{L}_{12}: \mathbf{L}_{01}: \mathbf{L}_{02}: \mathbf{L}_{03}\right]
$$

with $\mathbf{L}$ the Plücker matrix.

Given two planes $\pi_{0}=\left(\mathbf{n}_{0}, 1\right)^{T}$ and $\pi_{1}=\left(\mathbf{n}_{1}, 1\right)^{T}$, with $\mathbf{n}_{0}$ and $\mathbf{n}_{1}$ the normals of the planes, Plücker axial coordinates of their intersection are defined as:

$$
\mathcal{L}=\left[\mathbf{n}_{1}-\mathbf{n}_{0}: \mathbf{n}_{0} \times \mathbf{n}_{1}\right]^{T}
$$

where $\times$ is the cross product.

The projection of a $3 \mathrm{D}$ line $\mathcal{L}$ on a $2 \mathrm{D}$ line $l$ in $\mathcal{C}_{i}$ coordinate frame is written: 


$$
l_{\mathcal{C}_{i}}=f\left(\mathcal{L}, \mathbf{K}_{i}\left[\mathbf{R}_{\mathcal{C}_{i} \mathcal{W}} \mid \mathbf{t}_{\mathcal{C}_{i} \mathcal{W}}\right]\right)
$$

with

$$
\begin{aligned}
& \phi=\left\{\begin{array}{rr}
0 \text { if } l_{\mathcal{C}_{0}} \cdot x<0 \\
1 \quad \text { otherwise }
\end{array}\right. \\
& \Delta(i, \mathbf{n})=\left(\mathbf{I}_{0}\left[\mathbf{p}_{\mathcal{I}_{0}}^{i}\right]-\mathbf{I}_{1}\left[\mathbf{H}_{i}\left(\mathbf{p}_{\mathcal{I}_{0}}^{i}, \mathbf{n}\right)\right]\right)^{2}
\end{aligned}
$$
as seen in Figure 6.

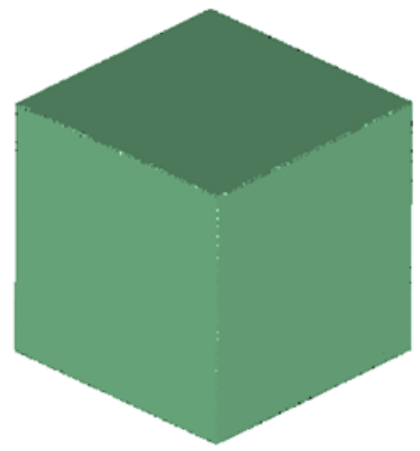

Fig. $63 \mathrm{D}$ reconstruction of the cube using the edge-optimized method (to be compared with figure 1) 


\subsection{Edge consistency}

The proposed edge model produces good results along sharp edges made of two faces. However, problems may occur when the edge model is used on surfaces with chamfers, spikes, small radii edges of fillets.

Both chamfers and spikes are composed of more than two planes. Small radii are not a surface discontinuity. In any case, our edge model cannot fit the surface perfectly. In the case of important noise on the surface, the algorithm may be biased and result in incorrect bi-plane fitting.

To prevent our method to include sharp edges in the model where they must not be or to accept inconsistent results we have added a verification step.

In order to discard wrong results produced by the edge optimization, we compare results obtained by the standard criterion to results obtained by the edge optimization and choose the best one. This works as both methods minimize a sum of squared differences on pixel intensity.

In addition we can set a threshold on the angle between the two-plane fitted by our algorithm to reject flat angles, that are mostly inconsistencies due to surface noise.

8 Results and discussion

The results presented in this section have been processed from images of a homemade stereo-vision system. It is composed of two $1024 \times 768$ CCD cameras with $8 \mathrm{~mm}$ lenses and a pattern projector. The stereoscopic baseline is $140 \mathrm{~mm}$ long and the cameras are oriented with a $15^{\circ}$ angle. The working distance is about $400 \mathrm{~mm}$ and the pixel size is about $0.23 \mathrm{~mm}$. During the image correlation process, $11 \times 11$ pixel correlation windows are used, leading to a spatial resolution of about $2.5 \times 2.5 \mathrm{~mm}^{2}$. The whole setup has been described and evaluated by Coudrin et al. [7]. The cameras and the projector are synchronized. We use a pattern projection to provide a dense 3D information from the pair of cameras, regardless 
of the texture on the object surface. When the system is triggered, the pattern is projected on the scene. Then the two cameras acquire images simultaneously. The pair of images is used in a reconstruction algorithm, providing a 3D point cloud by a stereo-vision surface reconstruction method. Points are expressed with respect to the frame $\mathcal{C}_{0}$ of the principal camera.

Figure 7 presents two 3D reconstructions of a mechanical part. The part contains both sharp and curved edges. On the left, one can see the result of the initial reconstruction. Sharp edges are rounded. On the right, one can note that the sharp edges have been corrected and are sharper. Curved edges have not been modified.
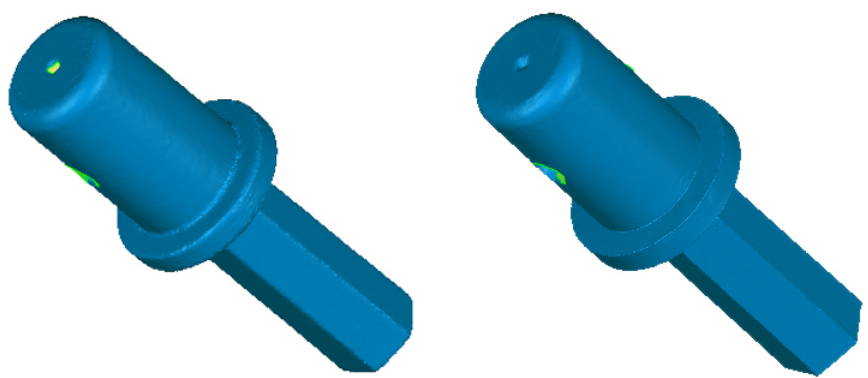

Fig. 7 Result of standard 3D reconstruction (left) and edge-optimized 3D reconstruction (right)

To illustrate the improvement of the new method, the deviation between the two results has been computed using Geomagic Qualify software (figure 8). The color map describes the signed euclidean distance between the two shapes and lies within the range $[-0.5 \mathrm{~mm},+0.5 \mathrm{~mm}]$. The largest errors can be observed along the edges. It shows that points on edges have moved up to $0.5 \mathrm{~mm}$ from their initial position.

Figure 9 shows a cylindrical gauge block. It is composed of a section of a cylinder with flat surfaces on the top and the bottom and perfectly circular surface 


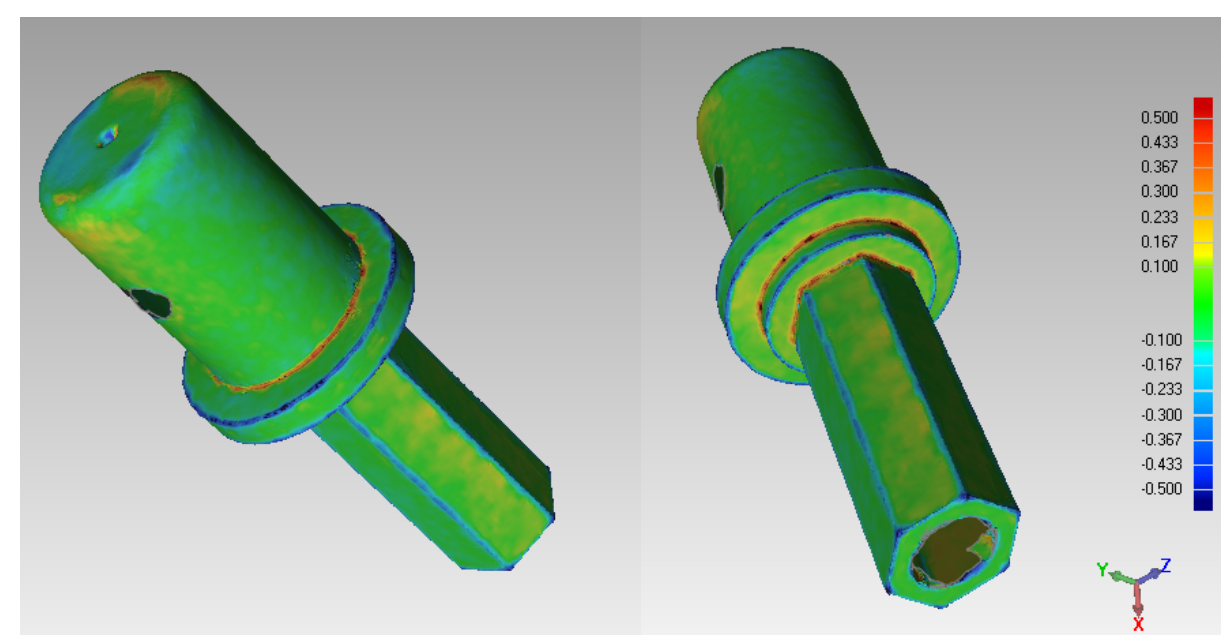

Fig. 8 Comparison between standard 3D reconstruction and edge-optimized 3D reconstruction. The color map represents the euclidean deviation between the two shapes

on the inside. The CAD model of this part is known and accurate up to $1 \mu \mathrm{m}$.

Figure 9 shows the initial reconstruction of the cylinder. Figure 10 presents the qualitative result of edge optimization. The edge at the intersection of the inner cylinder and the top surface has been sharpened. The other edges have not been altered since they are not actual sharp edges.

Since the CAD model is known for this part, we have evaluated the accuracy of our method by comparing the $3 \mathrm{D}$ reconstruction results to the theoretical surface. We have used the Geomagic Qualify ${ }^{\mathrm{TM}}$ software to align and compare our result to the CAD model ground truth. The software gives a comparison by projecting orthogonally each point of our 3D reconstruction to the surfaces of the CAD model. The result of the comparison is a distance map. Small projection distances are displayed in green, and tend to dark red or dark blue when the distance increases.

Figure 11 shows the distance map of the initial 3D reconstruction to the CAD model. The error increases strongly on the edge. Indeed, the initial reconstruction over-smoothes this sharp edge. Consequently, the points have been estimated far behind the actual surface. After the edge optimization process, Figure 12 shows 


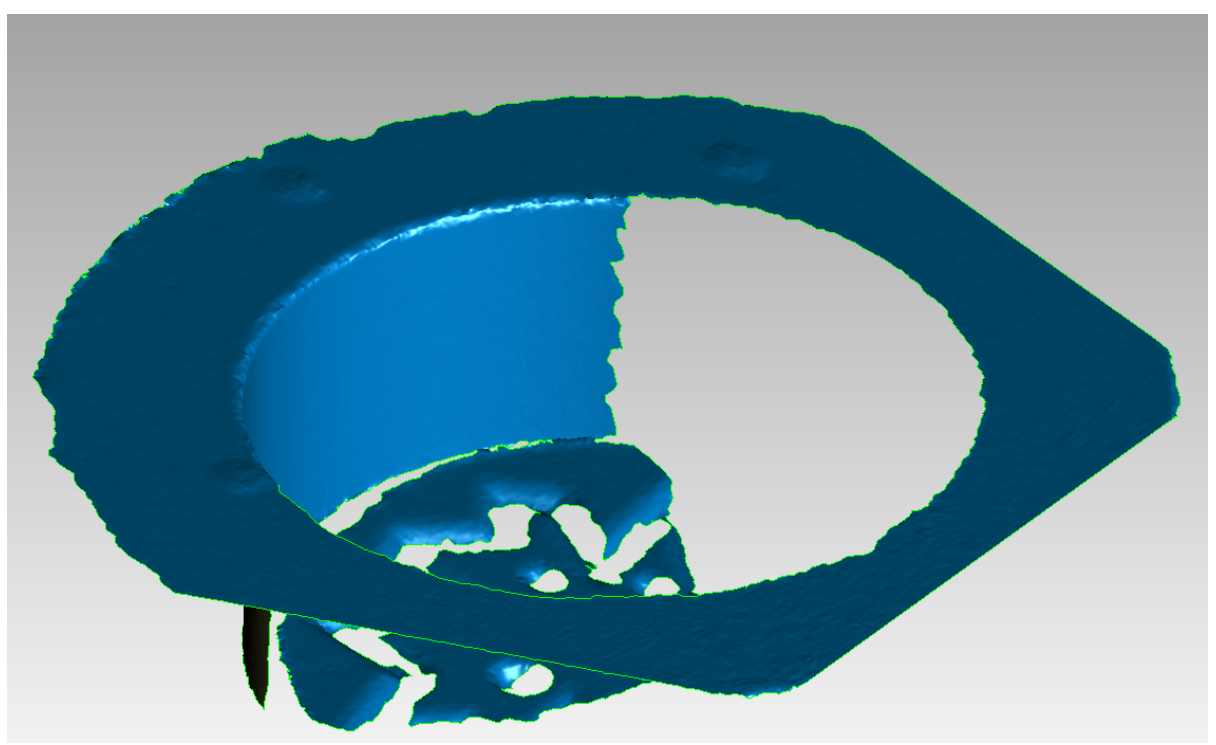

Fig. 9 Initial 3D reconstruction of a cylinder

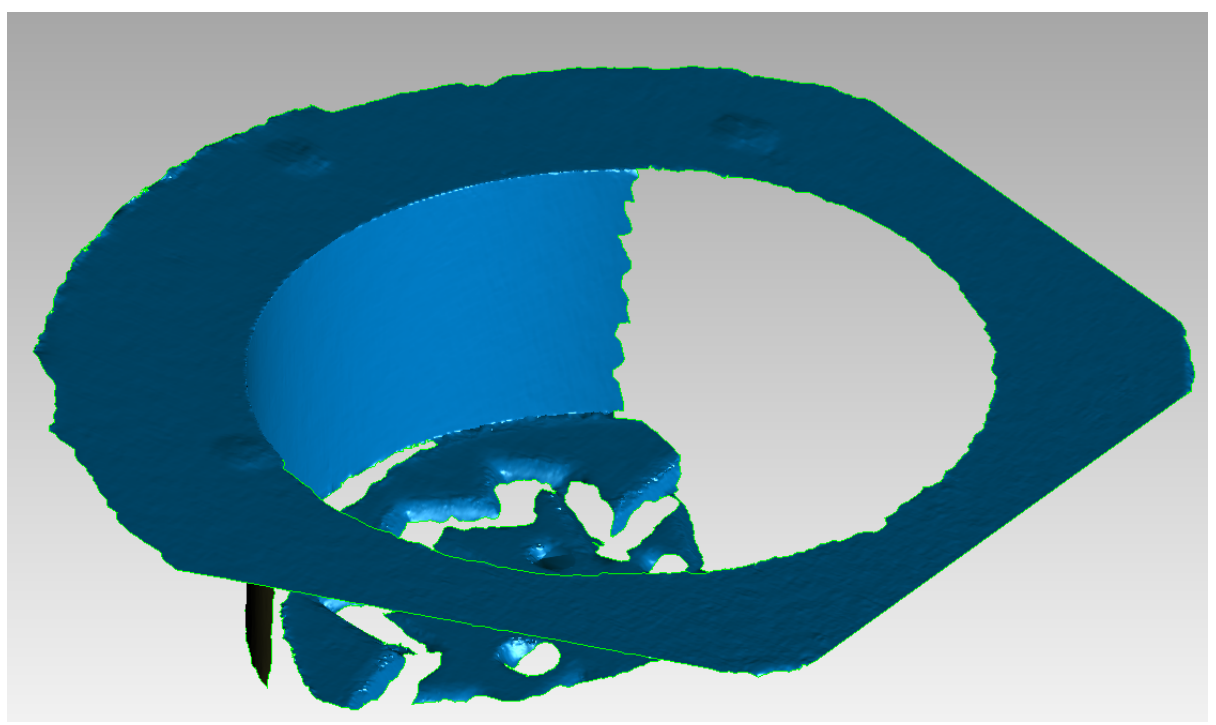

Fig. 10 Edge-optimized 3D reconstruction of the cylinder

the improved result. The edge has been accurately reconstructed and all the points on the edge are in the acceptable green range.

As a side effect of our method, one can note that the top surface contains a certain amount of error in figure 11 that is minimized in figure 12 . This is due to the 


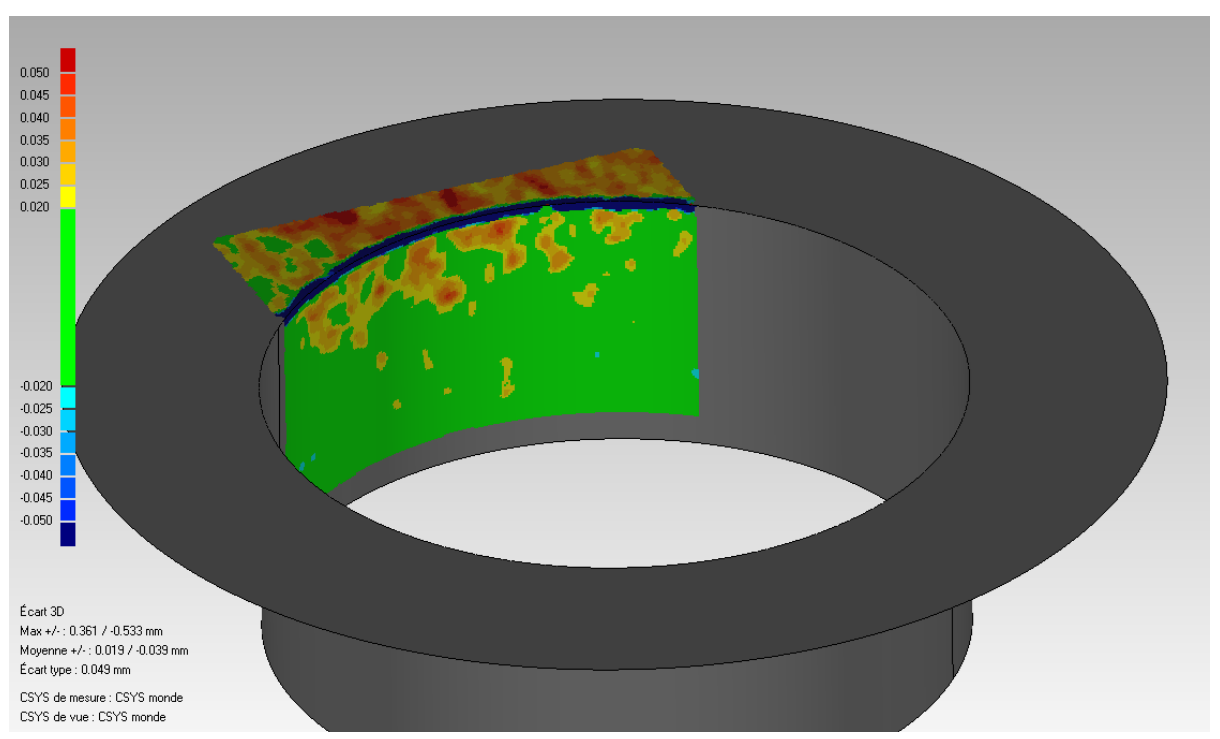

Fig. 11 Distance map of the initial 3D reconstruction to the CAD model

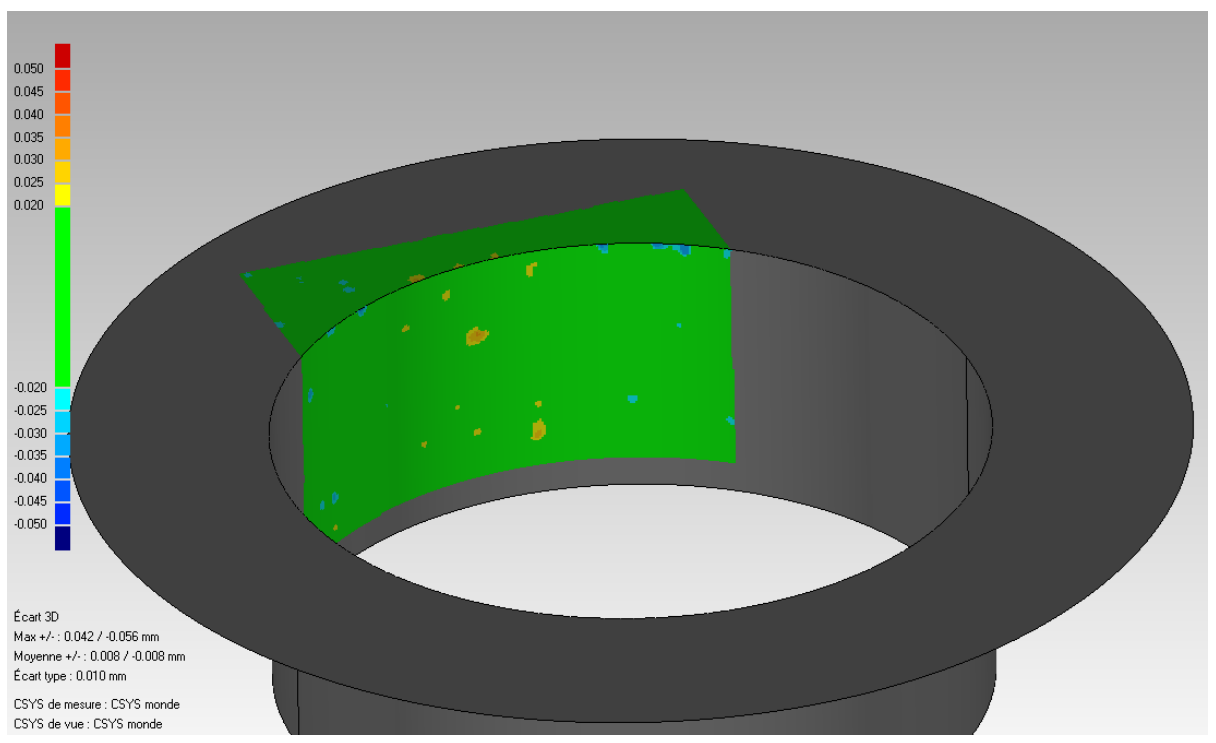

Fig. 12 Distance map of the edge-optimized 3D reconstruction to the CAD model

alignment step of the Geomagic Qualify software. Since the initial reconstruction contains more error, it is harder to align with the actual surface. With the edge improvement, the 3D reconstruction is more accurate and can be more easily

aligned to the CAD model. 
It should be noted that we have shown in [8] that the standard stereo-DIC approach does not perform well with a small window size $(3 \times 3$ or $5 \times 5$ pixel windows). Indeed, reducing the window size increases ambiguities in correlation and causes drifts in the stereo matching process, causing noise and holes in the 3D reconstruction. On the contrary, with a $11 \times 11$ pixel window, the noise is reduced and no holes appear, but the window becomes too large compared to the size of some small details, causing a smoothing effect (see Figure 10 in [8]). With the new multi-step stereo-DIC method proposed in this paper, a $11 \times 11$ pixel window can be used while reducing the smoothing effect.

\subsection{Accuracy comparison}

To give an absolute reference of the results provided by our method, we used a gauge block with a known geometry.

This part has been designed for measurement device calibration. It has been accurately manufactured and has an accurately known geometry. It is composed of several simple geometrical primitives (figure 13).

The ground truth CAD model (figure 14) is available and used to evaluate the reconstruction error

We compare our edge-optimized method to the standard pairwise stereovision approach. As we have seen previously, this approach tends to smooth details and edges. The gauge block is made of a large plane and geometrical primitives intersecting the plane, creating sharp edges. We also provide results obtained with the multi-view stereovision (MVS) method presented in [8].

Models resulting from the three methods (pairwise stereovision, edge sharpening in pairwise stereovision and our multi-view stereovision method) are registered on the CAD model of the gauge block. Points are projected orthogonally on the surfaces of the theoretical model and projection distance is measured for each point. This distance is considered as the reconstruction error of the point. Figure 15 represents these errors using a color map. Green areas are measured inside 


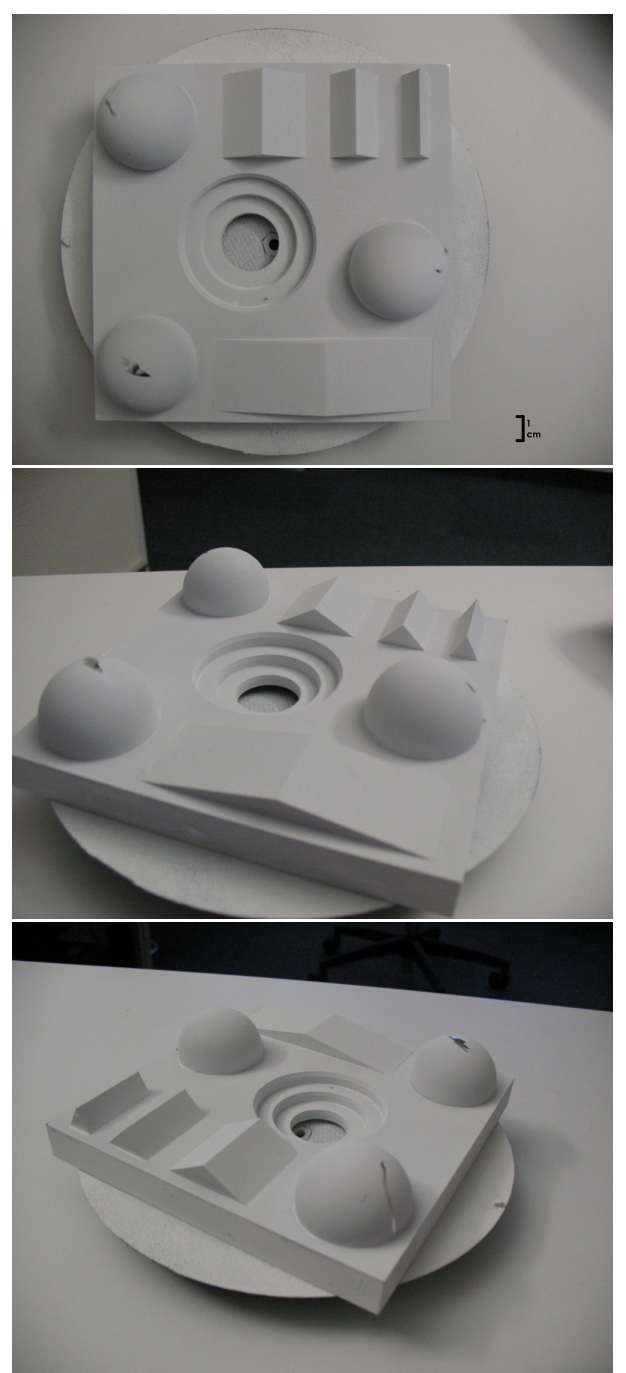

Fig. 13 The gauge block composed from simple geometrical primitives. It contains several sharp edges

the tolerance range $[-25 \mu m,+25 \mu m]$. Points with a larger positive error are represented on a scale from yellow to red. Points with a larger negative error are represented on a scale from light to deep blue.

As expected, the error map of the pairwise stereovision method shows a large error near edges of the model. Due to the smoothing effect of the surface approximation in the reconstruction process, edges are rounded. The error on the dominant plane is essentially contained in the tolerance range. The edge-optimized 


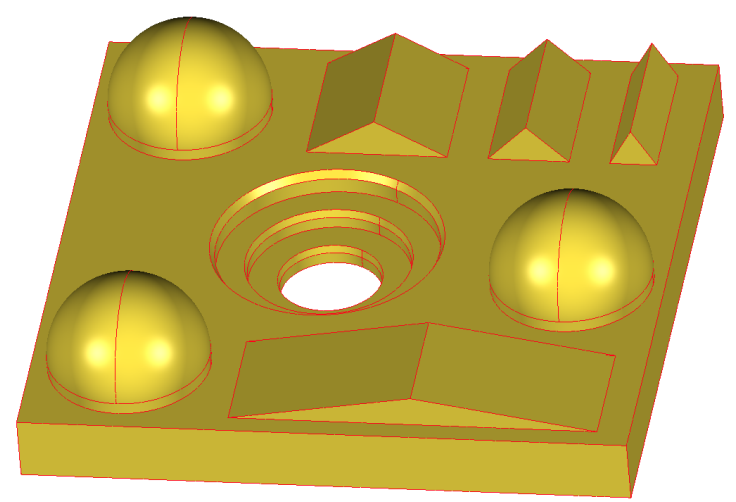

Fig. 14 The CAD model of the object is used as ground truth

\begin{tabular}{lrrr}
\hline & $\begin{array}{c}\text { Pairwise } \\
\text { stereo }\end{array}$ & $\begin{array}{c}\text { Edge } \\
\text { optimization }\end{array}$ & \multicolumn{1}{c}{$\begin{array}{c}\text { MVS } \\
\text { optimization }\end{array}$} \\
\hline Max error (mm) & 0.553 & 0.478 & 0.452 \\
Min error (mm) & -0.606 & -0.286 & -0.446 \\
Pos. mean (mm) & 0.036 & 0.037 & 0.023 \\
Neg. mean (mm) & -0.032 & -0.031 & -0.020 \\
Std. dev. (mm) & 0.066 & 0.049 & 0.033 \\
\hline
\end{tabular}

Table 2 Reconstruction error to the ground truth of the gauge block. Max/Min error : maximal positive and negative errors. Pos./Neg. mean : means of, respectively, positive and negatives errors. Std. dev : standard deviation on the error set

method performs a lot better along the edges. Maximal errors are now mainly located near complex edges - intersection of more than two surfaces - and along the chamfer of the central drilling. However, a small error on the edges remains. With our method, error is still mainly located near edges, but the model is more homogeneously inside the tolerance. The chamfers in the central drilling are now more finely reconstructed. It is to be noted that the dominant plane is also more finely reconstructed. A significantly larger part of the object is considered inside the tolerance comparing to the other methods.

Table 2 summarizes the results for this experiment. The results are presented showing maximal and minimal errors. The mean error should be $0 \mathrm{~mm}$, since the models are registered minimizing the distance between point clouds and the surface 


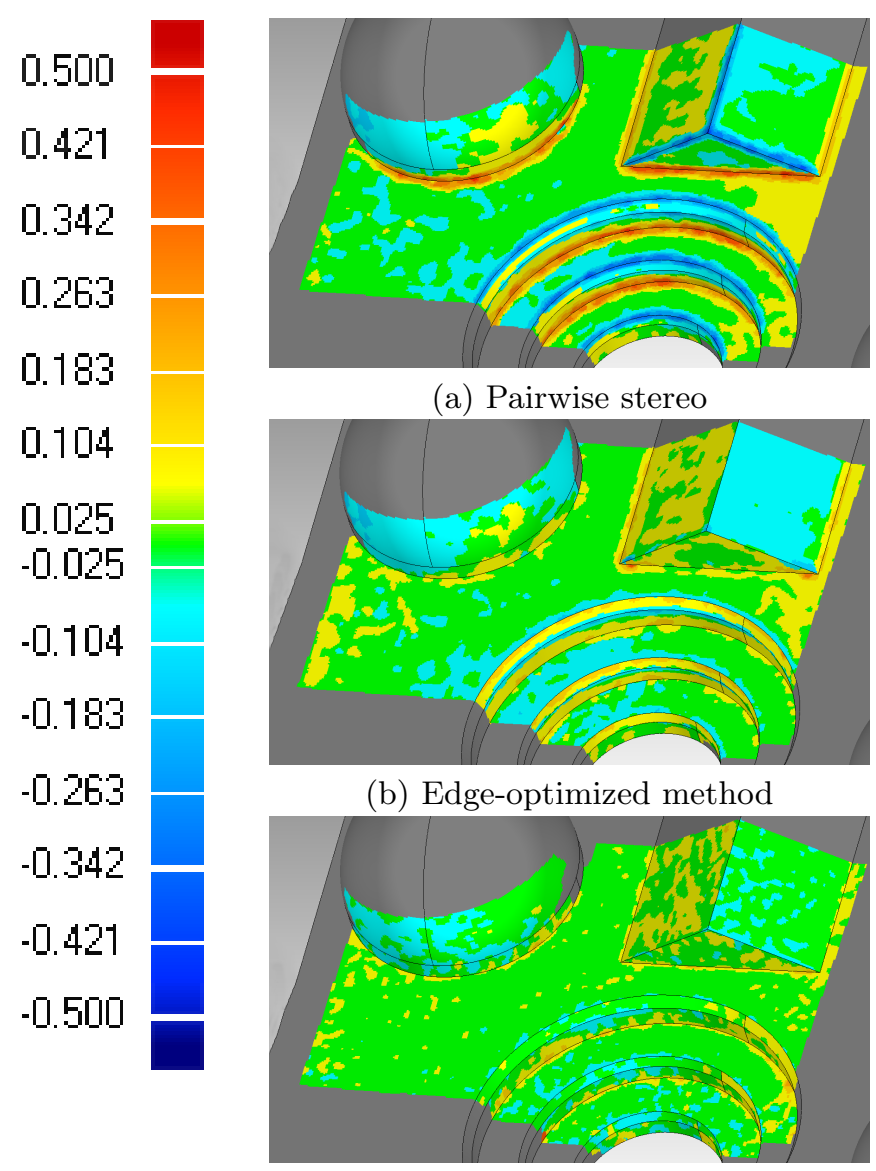

(c) MVS method

Fig. 15 Comparison of reconstruction error using pairwise stereovision, pairwise stereovision with our edge detection and correction method, and our multiview stereovision method

of the CAD model. It is then more meaningful to present average errors in positive and negative parts. The standard deviation on the error set is also presented.

As it was pointed in figure 15, our edge-optimized algorithm and our MVS method present better results and a higher accuracy in terms of deviation to the theoretical surface. The MVS method offers the best results, with the lowest deviation, but requires more images to be efficient. 
8.2 Discussion

We have introduced a new bi-plane model and a new correlation criterion in order to achieve a high accuracy $3 \mathrm{D}$ reconstruction along sharp edges. Standard windowbased methods suffer from the tradeoff between higher accuracy obtained with large subsets and more details obtained with small subsets. Our model permits to use larger subsets with the advantage of having both more details (reduced smoothing effect) and higher accuracy along the edges.

The main advantage of our method is that it performs well with a single stereo pair of images. Most of the state of the art methods which produce high accuracy $3 \mathrm{D}$ reconstruction rely on multiple views. Even if most of these methods perform better than our new bi-plane model, our method allows to improve the reconstruction along sharp edges when a multiple view configuration is not possible. This may be the case for those industrial applications where the number of cameras is limited or when the shape to be measured is not easily accessible.

The actual limitation of our method is that it only handles one kind of discontinuity: a bi-plane edge. With this model, more complex edges are not correctly modeled and are generally smoothed. Nevertheless, the general methodology described in this paper could be extended to handle more complex edge models (and associated correlation criterion) and a model switching mechanism could be implemented. By using the verification step described in section "7.2 Edge consistency" the model leading to the minimal correlation score could be kept locally. The choice of the best model could be even made easier in situations where the CAD model of the object to be reconstructed in 3D is available.

9 Conclusions and Future Work

We have developed a new multi-step stereo-DIC algorithm, based on a new correlation criterion and a new window transformation model, specially designed for measuring the 3D shape of machined objects with sharp edges. 
With a standard stereo-DIC-based shape measurement technique, sharp edges are generally smoothed. This effect can be minimized by choosing small size subsets but this increases the stereo matching uncertainty.

With our new method, reducing the subset size to avoid smoothing sharp edges is not necessary.

Results on real machined objects show that, with our new method, an accurate $3 \mathrm{D}$ reconstruction of the whole object, including the sharp edges that are preserved, can be achieved.

Many methods for 3D reconstruction are iterative and multi-resolution, improving an initial low-resolution model up to a representation with the required resolution and accuracy. Future work will be devoted to such a method, including using a prior knowledge provided by the object CAD model when available, in order to make simpler and more accurate the modeling process.

We also compared our method to a MVS method. Although the accuracy of MVS methods is better than our edge-optimized method, the latter requires only one pair of images and is able to produce honorable results. MVS methods require several images. It is legitimate to ask if combining both methods (MVS and edge optimization) and working with several images would improve the results.

DIC is widely used for shape and strain measurement. This work focused on shape measurement and we did not explore the eventual contribution of the edgeoptimized method to strain measurement. This will be part of further studies.

\section{References}

1. Pan B., Xie H., Wang Z., Qian K., and Wang Z. (2008) Study on subset size selection in digital image correlation for speckle patterns. Optics Express, 16(10):7037-7048.

2. Sutton M. A., Orteu J.-J., and Schreier H. W. (2009) Image Correlation for Shape, Motion and Deformation Measurements - Basic Concepts, Theory and Applications. Springer, ISBN 978-0-387-78746-6. 
3. Bornert M., Hild F., Orteu J.-J., and Roux S. (2012) Chapter 6: Digital image correlation. Grédiac M. and Hild F. (eds.), Full-Field Measurements and Identification in Solid Mechanics, pp. 157-190, Wiley-ISTE, ISBN 978-1-84821-294-7.

4. Reu P. (2012) Hidden components of 3D-DIC: Interpolation and matching - part 2. Experimental Techniques, 36(3):3-4.

5. Seitz S., Curless B., Diebel J., Scharstein D., and Szeliski R. (2006) A comparison and evaluation of multi-view stereo reconstruction algorithms. 2006 IEEE Computer Society Conference on Computer Vision and Pattern Recognition, pp. 519-528.

6. Furukawa Y. and Ponce J. (2010) Accurate, dense, and robust multi-view stereopsis. IEEE Trans. Pattern Anal. Mach. Intell., 32(8):1362-1376.

7. Coudrin B., Devy M., Orteu J.-J., and Brèthes L. (2011) An innovative hand-held vision-based digitizing system for 3D modelling. Optics and Lasers in Engineering, 49(910):1168-1176.

8. Harvent J., Coudrin B., Brèthes L., Orteu J.-J., and Devy M. (2013) Multi-view dense 3D modelling of untextured objects from a moving projector-cameras system. Machine Vision and Applications, 24(8):1645-1659.

9. Helm J. D. (2008) Digital image correlation for specimens with multiple growing cracks. Experimental Mechanics, 48:753-762.

10. Sjödahl M. (2010) Image and complex correlation near discontinuities. Strain, 46(1):3-11.

11. Poissant J. and Barthelat F. (2010) A novel "subset splitting" procedure for digital image correlation on discontinuous displacement fields. Experimental Mechanics, 50:353-364.

12. Pan B., Wang Z., and Lu Z. (2010) Genuine full-field deformation measurement of an object with complex shape using reliability-guided digital image correlation. Optics Express, 18(2):753-762.

13. Attene M., Falcidieno B., Rossignac J., and Spagnuolo M. (2003) Edge-sharpener: Recovering sharp features in triangulations of non-adaptively re-meshed surfaces. ACM Symposium on Geometry Processing.

14. Zhang Z. (2000) A flexible new technique for camera calibration. IEEE Trans. Pattern Anal. Mach. Intell., 22:1330-1334.

15. Hartley, R.I. and Zisserman, A. (2004) Multiple View Geometry in Computer Vision. Cambridge University Press, second edn. 